Nicole Brossard

\title{
Certains mots
}

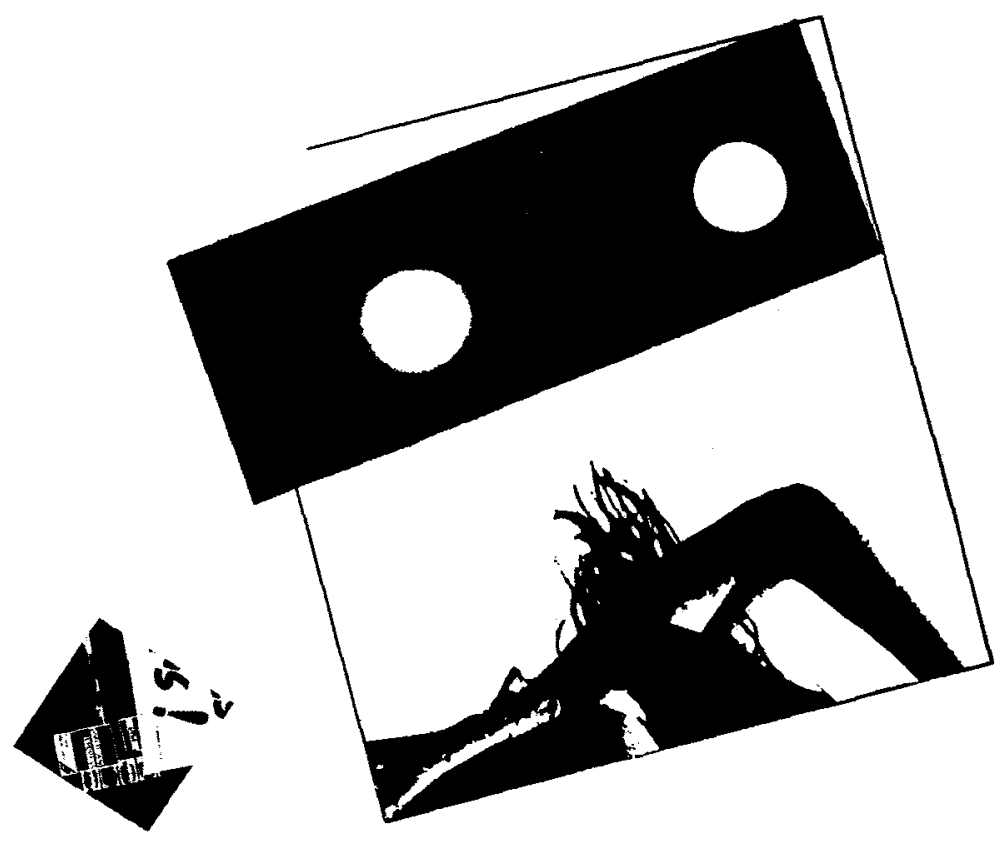


Parmi les pires malheurs, les plus audacieuses nuits d'adoration, la mort tragique, les peaux les plus douces, au bord des mers, vêtues d'un corps utopique et d'extases, nous avançons dans le relief des mots, habiles entre les coraux tranchants de l'Isla des Mujeres. Vêtues d'un corps de femme, nous patientons au bord des pages, attendant une présence féminine. Nous tournons les pages de nos doigts mouillés. Nous attendons que la vérité éclate.

D'une lecture à l'autre, les mots se relaient comme pour éprouver notre endurance autour d'une idée fixe, autour de quelques images que nous avons de nousmême et qui ne s'appliquent à nous qu'en l'espace fictif de la version que nous donnons de la réalité. D'une lecture à l'autre, nous fabulons autour de notre désir qui est d'identifier ce qui nous enthousiasme et qui nous plonge dans un tel état de ferveur «indescriptible».

Lorsque cette ferveur s'empare de nous, nous disons être captivées par notre lecture et nous avançons lentement/précipitamment vers notre destin. Notre destin est comme un projet, une vie tissée en nous par d'innombrables lignes dont certaines sont dites lignes de la main qui tient le volume. Ces lignes innervent notre corps tout entier comme une logique de la pensée des sens. En pleine lecture, nous reconnaissons (être) la cause et l'origine des visages et des paysages qui nous entourent car nous leur faisons allusion comme à une enfance, un désir, une tendance. En pleine lecture, 
nous entendons des murmures, des suppliques, des cris ; nous entendons notre voix qui cherche son horizon.

Il y a dans nos lectures des mauves, de l'indigo, des regards terribles, des femmes vêtues de bijoux et de silence. Il y a des corps éprouvés. Il y a de troublantes apparitions. Nous ouvrons et refermons les yeux sur elles dans l'espoir d'une suite sonore ou d'un entretien. Toute notre ferveur s'engouffre dans cet entretien afin qu'éclate la vérité.

Parmi la rhétorique, la logique des sens, les paradoxes et la sensation de devenir, nous avançons dans l'intention des formes. Il arrive qu'en pleine nuit nous nous éveillions pour relire un passage et revoir des femmes que nous désirons. Et pendant que nous relisons le passage souligné, il y a dans notre poitrine une sensation «indescriptible» qui nous garde éveillées jusqu'à l'aube. À l'aube, notre esprit est extravagant ; il erre dans des zones interdites et nous n'avons d'autre choix que de les explorer. On m'a dit que des femmes écrivent à l'aube lorsqu'elles sont dans cet état. On m'a dit que parfois elles éclatent en sanglots.

"Je connais les rythmes de la voix, je connais ses sursauts. Je connais l'aventure et l'expérience du regard». C'est ce vers quoi nous nous élançons à chaque lecture, incrédules devant la vérité qui éclate en nous comme une mémoire de l'ombre et de la ferveur.

Les mots que nous remarquons s'appliquent à nous et nous remplissent d'inquiétude et de plaisir. Ces mots sont révélations, énigmes et adresses. Nous les transformons selon une méthode d'approche qui échappe à notre conscience et pourtant notre conscience s'en trouve éclairée. Lectrices, nous devenons l'allusion et la 
tendance d'un texte.

Ce qui nous anime en une phrase ou une expression est une décision de l'être. Alors c'est à toute allure que nous nous penchons sur le texte afin d'atteindre dans le feu de l'action la geste de notre désir. Chaque lecture intense est un rayon d'action dans lequel nous surprenons sur le fait l'unanimité qui se fait en nous.

Parmi les axes, les équations, les audaces enivrantes et la lumière que nous croisons, nous avançons dans notre lecture comme en théorie nous devenons ce que nous désirons. Nous avançons vers une femme subtile et complexe qui reflète le processus et les formes du développement de notre pensée. Les mots sont une manière de dévorer le désir qui nous dévore avec des comparaisons qui nous amènent là où nous devenons l'appétit de la science et la science de la conscience.

Lorsque de nos doigts mouillés, nous tournons les pages, allant de la frayeur à l'extase, nous affrontons l'éternité crédules et athées devant la somme des corps, des crânes, des orgasmes; nous affrontons l'au-delà de l'ensemble et nous devenons la précision du désir dans l'espace inénarrable du cerveau.

Vraiment, l'effet sensationnel de la lecture est une sensation que nous ne pouvons pas exprimer, sinon qu'en soulignant. L'intime de l'éternité est une intrigue que nous inventons à chaque lecture. Toute lecture est une intention d'images, une intention de spectacle qui nous donne espoir. 


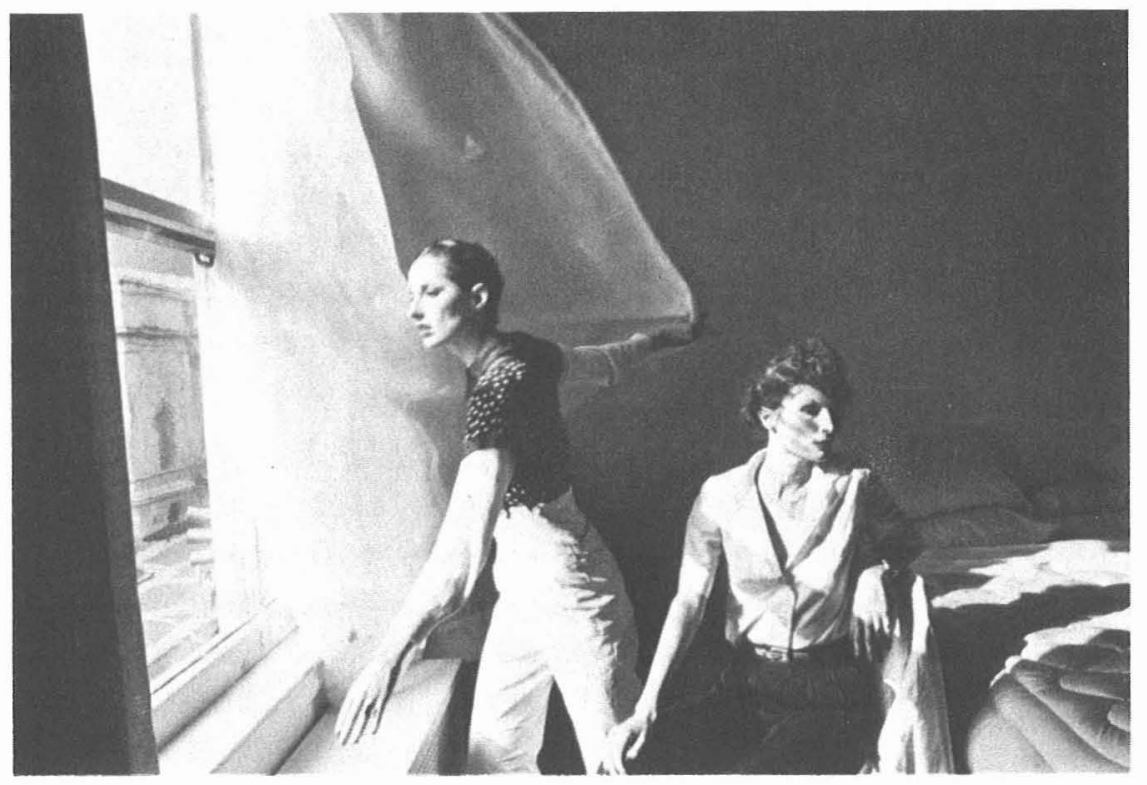

CERTAIN WORDS MUST BE SAID. 1970

PHOIOGRAPH BY DUANE MICHALS

Clanis,

l'ai passi' la nus't à line ton dernies livere. 9'en ai fait mom lives. Sl y a tellament d'annotations que personne d'autre que mori me pouna se cenvis de liselmplair que to m'a adressi. Ye suis daves l'inca. pacite' de to drie pricise'ment en quoi j'aims ce livins. $9 l$ me sera cependaut possiste d'en facie une critigue poun le mois d'octorse. Oe vis a jaradoxe comms unes itrias. gete' dus sadoir, un malai'se d'iciture.

Love hinote

(C) 1980 DUANE MICHALS
PRINIED BY RAPOPORT PRINIING CORP

(C) FOIOFOLIO, BOX GOI CANAL STA. NY. NY 10013 
Claire Texte vient de publier un des plus beaux livres que nous ait donné notre littérature. Dans un récit de

l'improbable affirmation .rien à changer

un propos d'existence

La beauté de l'écriture

Ces paysages au bord de la mer............. descriptions.... un effet mécanismes

allusion. Il faut lire 
image. La soif est au corps un délire. Tout s'embrouille autour de la piscine suivi d'un long monologue de la rousse qui raconte son enfance «Écoute, écoute-moi bien» puis il y a vingt pages écrites à la première personne sur un ton familier. Le passage serait facile à traduiresauf pour quelques mots pourtant simples comme eau, chaleur, poitrine. C'était une question d'atmosphère. Il lui faudrait tourner autour de l'expression «ma mère» et par une ruse de syntaxe faire précéder le mot poitrine de "très jeune» accompagné du verbe être ; il faudrait aussi faire en sorte qu'à la

\} lecture l'attention puisse se porter simultanément sur la voix et le bijou en forme de papillon «lorsqu'elle interrompait sa lecture pour me dire il fait nuit maintenant». Les pages défilaient dans le temps, la famille, une chasse à l'homme sur des autoroutes qui suivaient l'horizon, le bruit des sirènes puis un état d'âme, une inquiétude et la passion qui se transforme en des signes, un tatouage sur l'épaule, et les excès. Elle était ailleurs.

Dans le flamboiement des mots à toute vitesse.

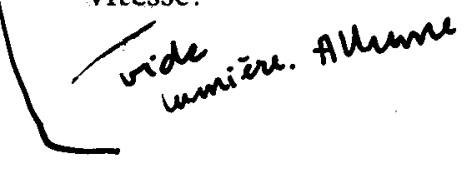

